\title{
Kernos
}

Revue internationale et pluridisciplinaire de religion grecque antique

4 | 1991

Varia

\section{Les kernoi éleusiniens}

\section{Georges Bakalakis}

URL : http://journals.openedition.org/kernos/293

DOI : $10.4000 /$ kernos.293

ISSN : 2034-7871

\section{Éditeur}

Centre international d'étude de la religion grecque antique

\section{Édition imprimée}

Date de publication : 1 janvier 1991

Pagination : 105-117

ISSN : 0776-3824

Référence électronique

Georges Bakalakis, « Les kernoi éleusiniens », Kernos [En ligne], 4 | 1991, mis en ligne le 11 mars 2011 , consulté le 30 avril 2019. URL : http://journals.openedition.org/kernos/293 ; DOI : 10.4000/kernos.293 
Kernos, 4 (1991), p. 105-117.

\section{LES KERNOI ÉLEUSINIENS}

Bien que la kernophoria n'ait pas à l'origine appartenu au cérémonial du culte initiatique des divinités éleusiniennes, les vases de forme particulière et de structure composée qu'ont livrés en grand nombre d'une part la fouille du sanctuaire d'Éleusis et de l'autre celle de dépôts sur le site de l'antique agora d'Athènes, qui proviennent de l'ḱv $\alpha$ $\sigma \tau \varepsilon \iota$ 'E $\lambda \varepsilon v \sigma i v i o v$, se retrouvent dans la description minutieuse du kernos que fait Athénée (XI, 478d) d'après l'Ilion de Polémon et le témoignage d'Ammonios (qui a vécu au Ier s. ap. J.-C.) ${ }^{1}$.

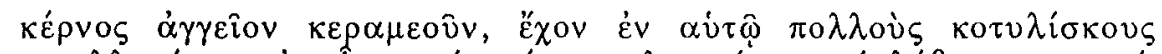

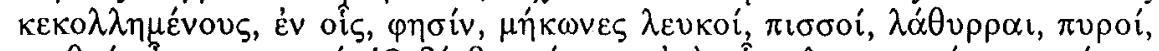

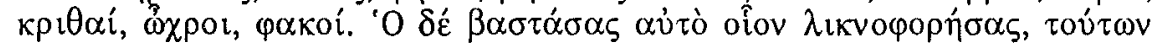

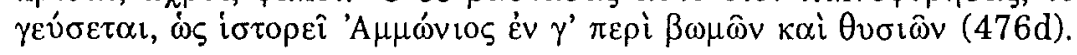

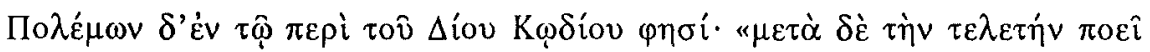

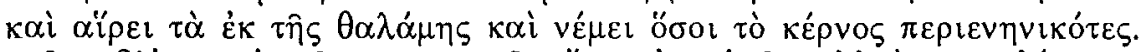

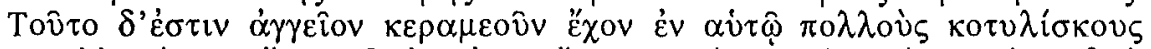

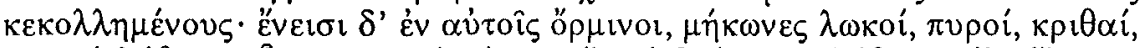

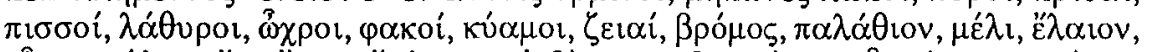

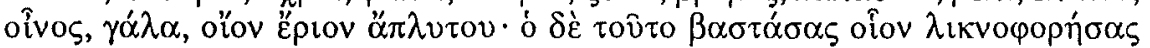

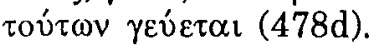

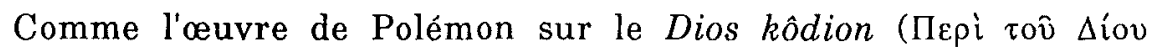
$\kappa \omega \hat{\delta}$ íov), mais encore le kôdion lui-même sont associés aussi au culte éleusinien, les divers savants qui ont étudié le sujet ont supposé que la description du vase de même que la pratique de la kernophoria concernaient le culte éleusinien.

En outre, quoique initialement considéré comme un récipient sacré du culte des divinité chthoniennes Rhéa et Cybèle, le kernos se rapporte indirectement au culte de Déméter aussi, puisque le Dion kôdion jouait un rôle important dans le culte éleusinien ${ }^{2}$. De surcroît, on n'a trouvé nulle part ailleurs autant de kernoi que dans le sanctuaire d'Éleusis et

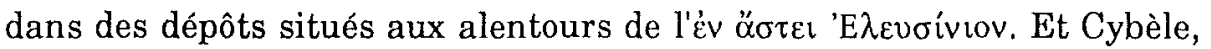
cette divinité d'origine étrangère dont le culte au Pirée remonte au Ve s.

1 Cf. O. Rubensohn, Kerchnos, in MDAI(A), 23 (1898), p. 275; J. Pollitt, Kernoi from the Ancient Agora, in Hesperia, 48 (1979), p. 205 sq.

2 Bien que la scholie du Gorgias de Platon (493c) ait pu faire l'objet de contestation. Cf. PolLiTT, art. cit., p. 206, n. 4. 
av. J.-C. et qui fut également honorée dans le métrôon de l'agora, s'apparente d'une manière évidente à Déméter ${ }^{3}$.

À l'époque classique, le kernos se rapporte généralement au culte des divinités chthoniennes Rhéa et Cybèle, mais les faits enseignent que, tout au moins ultérieurement, il était associé d'une certaine façon aussi au culte de Déméter. Quoi qu'il en soit de l'utilisation première et de la signification du kernos, la kernophoria a été adoptée par le cérémonial éleusinien, puisque, hormis l'abondance de kernoi trouvés dans le sanctuaire, la représentation d'une kernophoria sur la célèbre plaque en terre cuite de Niinnion nous l'atteste 4 .

Nous préciserons enfin que les vases provenant des sanctuaires

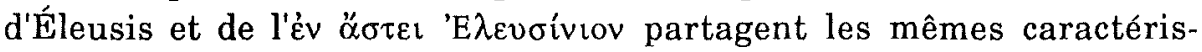
tiques morphologiques et qu'ils sont les seuls à mériter l'appellation de kernoi éleusiniens, tandis que tous les autres, trouvés ailleurs dans l'aire grecque, même s'ils sont plus anciens que ceux-là, doivent être appelés tout simplement kernoi $i^{5}$. Le matériel le plus fragmentaire des fouilles de l'École américaine d'Études classiques a été publié intégralement par notre confrère J. Pollitt ${ }^{6}$. La publication annoncée de tous les kernoi du Musée d'Éleusis que nous élaborons se présentera comme un corpus et rassemblera également les archives de K. Kourouniotis en préparation depuis de longues années. Que l'on ne voie donc dans ces quelques lignes qu'un souhait de bienvenue au nouveau périodique qui porte le nom de kernos, en tant que calice destiné à recueillir les fruits de l'étude de la religion grecque antique.

La forme fondamentale du kernos éleusinien est celle d'un cratérisque aux caractéristiques suivantes. Du pied, conique et concave, s'évase la panse, divisée horizontalement en deux parties par l'antyx

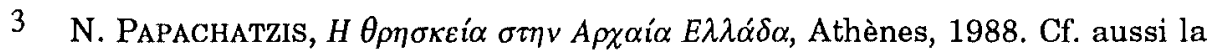
statue de la Mère des Dieux à l'agora d'Athènes, œuvre de Phidias ou

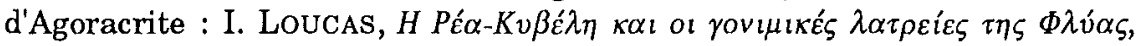
Athènes, 1988, p. 15-22, où il est question de l'introduction du culte métroaque à Athènes.

4 Cf. G. MYlonas, Eleusis and the Eleusinian Mysteries, Princeton, 1961 (1974), fig. 88.

5 C'est aussi la distinction faite par F. SCHAUROTH-UPSON dans sa dissertation non publiée intitulée The Kernos in Ancient Cult, Redcliff College-Harvard University, 1942, p. 44 sq. (les kernoi éleusiniens) et p. 122 sq. (le kernos dans l'initiation éleusinienne). Cf. aussi PolLiTT, art. cit., p. 228. 
(pour ce terme, cf. infra) et l'épaule arrondie s'achève sur une bouche proportionnellement large. La base du pied, la ligne de rupture entre ce dernier et la panse, ainsi que la bouche sont souvent soulignées horizontalement de cordons. Des anses horizontales naissent du bord inférieur de l'antyx tandis que sur son bord supérieur les cupules (kotyliskoi) s'alignent en une ou plusieurs rangées. L'antyx est percée transversalement de quatre petits trous disposés en croix sur son pourtour, où était piquée une branche de myrte. Le bas du pied comporte, lui, deux trous d'un diamètre plus large, opposés l'un à l'autre, où passait la lanière avec laquelle le kernos était fixé sur la tête.

La présence de l'antyx est doublement justifiée. Elle sert de support aux cupules qui peuvent couvrir presque la totalité de la partie supérieure du vase, lorsqu'elles sont disposées en plusieurs rangées, mais encore, sur le plan technique, elle fait office de joint, de jante (en anglais, flange), comme les anneaux métalliques, appelés brides, qu'utilisent aujourd'hui les plombiers pour assembler deux tuyaux. Car le vase était fabriqué au tour ou au moule en deux partie séparées. Nous avons ainsi préféré le terme antyx à stephanis qu'a choisi $\mathrm{K}$. Kourouniotis, ou celui de Randstreife dont use O. Rubensohn.

D'après ces principales caractéristiques, et essentiellement suivant qu'il y a ou non des cupules issant de l'antyx, nous classifions les kernoi éleusiniens en quatre types. J. Pollitt en dénombre également quatre, mais notre classification se différencie toutefois de la sienne sur plusieurs points.

Le type A du grand kernos avec huit grandes cupules n'est pas uniquement représenté par la trouvaille d'Éleusis ( ${ }^{\circ} \mathrm{A} .1$ du corpus des kernoi en préparation, fig. 1 des illustrations de cet article) mais aussi par des trouvailles de l'agora ${ }^{7}$. Ce vase ${ }^{8}$ offre du point de vue de la forme une ressemblance avec les kernoi non éleusiniens, mais cela ne signifie pas pour autant que, parmi les kernoi éleusiniens, ce type soit le plus ancien.

Les récipients de ce type, d'une grande capacité partagée entre les cupules et la panse, peuvent contenir une importante quantité des divers produits de la terre que mentionne Athénée.

7 PoLLITT, art. cit., pl. 65 b.

8 Hauteur (reconstituée) : $13 \mathrm{~cm}$; diamètre d'une anse à l'autre : $21 \mathrm{~cm}$; diamètre de l'ouverture : $7,5 \mathrm{~cm}$. 
Dans certains de ces kernoi, les cupules se réduisent de plus en plus, jusqu'à devenir schématiques et symboliques. Aussi les biens offerts à l'occasion de la kernophoria ne remplissent-ils que la panse, tandis que les cupules symbolisées se répartissent en une ou plusieurs rangées qui s'échelonnent sur l'épaule du kernos. L'accent est mis sur les deux anses qui s'infléchissent vers le haut du vase et soutiennent une large cupule. Chacune de ces cupules pouvait contenir une certaine quantité d'offrandes, mais il est également possible qu'elles aient joué le rôle de chandeliers ou qu'elles aient servi de lampes, comme le laisse entendre

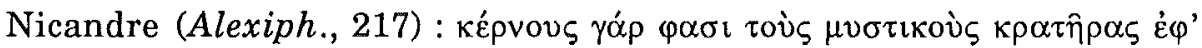

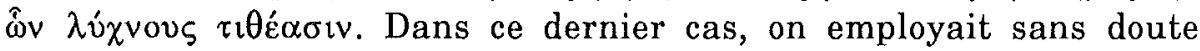
comme mèche la fleur séchée de la Ballota acetabulosa, plante qu'aujourd'hui les Grecs appellent loumini - mot qui signifie "petite mèche".

On a donc ainsi pris connaissance du type B du kernos éleusinien bien représenté par le $n^{\circ}$ B. 6 de notre corpus en préparation et qui correspond ici à la fig. $2^{9}$.

Nous choisissons le kernos $n^{\circ}$ B.22 (fig. 3) pour illustrer d'une manière représentative les kernoi du type $\mathrm{B}$ à cupules symboliques disposées en rangées successives. Sur ce vase, hormis les trois séries de cupules d'une certaine profondeur, on remarque sur une anse (l'autre a disparu) un petit pain rond moulé, semblable à celui du relief de Vesta à Berlin ${ }^{10}$. On se souviendra également du palathion dans le texte d'Athénée. Souvent les petits pains sont encore plus simplifiés et prennent l'aspect de graines de lentille, et plusieurs sont même pourvus d'une pointe saillante afin de mieux adhérer à la paroi du vase ${ }^{11}$. Ils ressemblent aux koulourakia, ces petits gâteaux généralement ronds de la Grèce moderne et il ne fait aucun doute que leur représentation est symbolique. Ils sont figurés en une rangée sur l'antyx, ou en deux ou. encore plusieurs rangées, sur l'antyx et la naissance de l'épaule convexe; le plus remarquable est qu'ils sont placés à intervalles réguliers de quatre petits pains de la rangée intérieure, tandis qu'ils dissimulent le cinquième : comment ne pas se souvenir à ce propos des

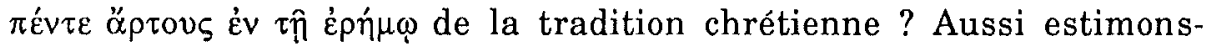

9 H. : 11,5 cm; diam. aux anses : $27 \mathrm{~cm}$; diam. ouv. : 10,5 cm. Certaines parties de la base du vase, une cupule et une anse sont reconstituées.

10 Cf. A. Greifenhagen, Das Vestarelief aus Wilton House, Berlin, 1967 (B.W.Pr., t. 121-122), p. 18, fig. 9, 11-12, pl. I.

$11 \mathrm{H}$. conservée : $5,5 \mathrm{~cm}$; diam. à l'antyx : 15,5 cm. Le pied s'est détaché. 
nous que le jugement qu'émettait $\mathrm{O}$. Rubensohn ${ }^{12}$ selon lequel ces représentations nun rein ornamental verwandt wurden ne tient pas.

L'exemple choisi pour illustrer cette catégorie est le kernos B.23 (fig. 4) ${ }^{13}$, reconstitué pour une grande part, mais sans erreurs ${ }^{14}$.

Parmi le type $\mathrm{C}$, nous avons finalement classé les quelques exemples qui rappellent le type $B$ en ce qu'ils sont pourvus de petits et de grands trous scrupuleusement répétés ainsi que d'anses horizontales, mais s'en distinguent par contre par la poignée en anse de panier qui se développe depuis la bouche du kernos ${ }^{15}$.

Les kernoi, dont les cupules étaient la caractéristique, ne présentent finalement que l'antyx, les anses horizontales plus ou moins atrophiées, les petits ou grands trous, mais pas de cupules. Et ce sont les plus nombreux, non seulement à Éleusis, mais aussi au Musée de l'agora dans la publication du professeur J. Pollitt. Dans notre classification, ils sont désignés par la lettre $D$.

Cela peut paraître paradoxal de parler de kernoi à propos du type $\mathrm{D}$, et pourtant ce n'est pas injustifié. Les kernoi figurés sur la plaque de Niinnion n'ont pas de cupules. À une époque ultérieure et après l'usage du vase dans le culte éleusinien, à la fin du IVe s. av. J.-C. et aux siècles suivants, la figuration des kernoi dans différentes marques et divers symboles monétaires, sur des monuments en ronde-bosse, en bronze et en plomb, hormis le relief classicisant du hiérophante Agnousios, est dénuée de cupules.

Les quelques kernoi en bronze du Musée d'Éleusis, de même que ceux de marbre, à considérer comme dédicatoires par excellence, n'ont pas de cupules non plus. Ainsi s'intègrent-il également au type D. La fouille sur le site de l'agora, quant à elle, n'a pas livré de kernoi de bronze ou de marbre.

Les kernoi en céramique du type $\mathrm{D}$ à Éleusis se divisent en divers sous-groupes : les non vernissés, ceux qui sont recouverts d'un enduit blanc pareil à celui des lécythes à fond blanc, mais ne sont pas d'une aussi bonne qualité quant à l'épaisseur, ceux qui sont décorés de

12 Rubensohn, art. cit., p. 284, pl. XIII, 5-6; cf. aussi ibid., p. 283 : «zu einer veritablen Rosette vereinigt sind".

13 Cf. le dessin du vase dans RuBENSOHN, art. cit., pl. XIII, 5.

$14 \mathrm{H}$. conservée : $16 \mathrm{~cm}$; diam. à l'antyx : $23 \mathrm{~cm}$.

15 L'ancien numéro d'inventaire de ce kernos en grande partie reconstitué est 2195.19. H. conservée (l'anse exclue) : $12,6 \mathrm{~cm}$; diam. à l'antyx : 13,5 cm; profondeur : $8,5 \mathrm{~cm}$. 
branches de myrte à neuf feuilles ou d'épis de blé, en cinq points de l'épaule du kernos (fig. 6) ${ }^{16}$.

Un autre sous-groupe comporte sur l'épaule une figuration très simple d'un motif linéaire à identifier aux anneaux qui ceignent les faisceaux - bakchoi - que tiennent les mystes sur diverses représentations d'initiés mythiques ${ }^{17}$. Dans les figurations plus anciennes des vases, la polychromie apparaît : du rouge, du vert, du blanc, lorsque tout le vase n'est pas blanc ${ }^{18}$. Certains kernoi du type D comportent une dorure sur l'épaule, sans que cela signifie qu'il y ait un rapport

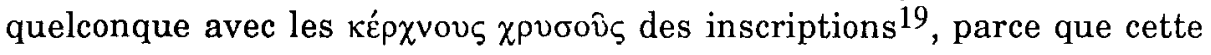
mention d'objets précieux et décoratifs qui appartiennent aux déesses éleusiniennes ne désigne pas des vases - kernoi - en or, mais des bijoux confectionnés selon la technique de granulation ${ }^{20}$. De toute façon, indépendamment de la signification exacte des кé $\chi \chi v o \imath$ des inscriptions, puisque nous avons à Éleusis une quantité de kernoi en terre cuite, en bronze et en marbre, l'étude prématurée et partielle qu'en a faite $O$. Rubensohn ne perd rien de sa valeur scientifique, à

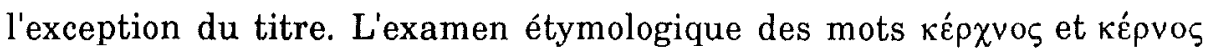
sort cependant du cadre de cet article.

Les kernoi de la fouille de l'agora, bien qu'ils ne proviennent pas

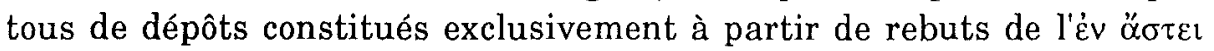
'E $\lambda \varepsilon v \sigma i v i o v$, sont datés d'après le contexte archéologique dans lequel ils ont été retrouvés (principalement des tessons de céramique figurée ou à vernis noir) du Ve s. av. J.-C. à la fin du IVe s. av. J.-C. Il est même un fragment à dater aussi tard que le IIIe s. av. J.-C. ${ }^{21}$

16 L'ancien no inv. 106. Diam. à l'antyx :7,2 cm; diam. d'une anse à l'autre : $10 \mathrm{~cm}$.

17 Cf. par ex. MYLoNAS, op. cit., fig. 78.

18 Cf. $A E(1885)$, p. 171 sq.

19 I.G., $\mathrm{I}^{3}, 386,17$ (408/407 av. J.-C.); 390, 8 (ca $420-405$ av. J. -C.) = I.G., $\mathrm{I}^{2}, 313$, $314,317$.

20 H.G. PRINGSHEIM (Archäologische Beiträge zur Geschichte des eleusinischen Kults, Munich, 1905, p. 69-72) et F. LEONARD (s.v. Kernos, in RE, XI, 1 [1921], p. $316 \mathrm{sq}$.) n'ont pas accepté l'équivalence $\kappa \varepsilon ́ p \chi v o \varsigma=\kappa \varepsilon ́ p v o \zeta$ et récemment Tullia LINDERs (Kerchnos and Kerchnion. Not Kernos but Granulation, in OAth, 17 [1988], p. 229 sq.) est revenue sur le sujet avec plusieurs catalogues d'objets provenant de divers sites.

21 PolditT, art. cit., p. 26, pl. 70,6. 
Les kernoi d'Éleusis proviennent tous de la fouille du vénérable sanctuaire ${ }^{22}$ et précisément de celle conduite par D. Philios et A. Skias aux trois endroits suivants : 1) «tout près du Télestérion ou pas tellement loin de ce dernier; plus précisément, $D$. Philios, qui est le premier à avoir trouvé de tels vases, mentionne comme lieu de découverte un endroit devant le portique de Philon (317-307 av. J. -C.), à courte distance de la façade et dans une couche qui comportait de fortes traces d'incendie, à une profondeur de 2 à $2,50 \mathrm{~m}$ sous le niveau de sol du portique de Philon, soit de 1 à $1,50 \mathrm{~m}$ sous le dallage romain de la cour du Télestérion, entre les repères $\rho$ et $\sigma$ du croquis de la fouille publié dans les $P A E$ de 1882 » $23 ; 2$ ) «dans le remblai entre l'angle nord-est du temple (Télestérion) et le mur de briques crues où se trouvaient les anciens silos »; 3) «sous le niveau du sol du Bouleuterion, on a dégagé d'une part dans les couches supérieures une grande quantité de vases éleusiniens particuliers, dont plusieurs étaient dorés (...); d'autre part, toutes les terres sous le sol du Bouleuterion semblent avoir été déversées expressément et en une fois pour remblayer" 24 . D'après K. Kourouniotis et I. Travlos, «au cours de la fouille effectuée en 1938 pour dégager et examiner la face arrière du mur sud (...) sur une longueur de $4 \mathrm{~m}$ environ et sur une largeur d'1 m, nous n'avons pas progressé en profondeur de plus de 2 mètres (...). Dès le début de la fouille, nous avons trouvé les kernoi, et on a continué d'en découvrir tout au long de la fouille près de l'escalier du bâtiment de surveillance, dans la cour de celui-ci» 25 .

Ainsi aucun kernos parmi ceux qui se trouvent au Musée d'Éleusis n'est plus récent que la moitié du IVe s. av. J.-C. L'expression de D. Philios "sous le dallage romain de la cour du Télestérion" a été mal comprise et a facilement fait l'objet d'une interprétation erronée de la part de 0 . Rubensohn ${ }^{26}$ et de Leonard ${ }^{27}$ qui parlent de kernoi d'époque romaine qui auraient été découverts sous le dallage de la cour, alors que personne n'aurait eu l'idée d'y fouiller ou que personne n'aurait pu

22 Pour une bibliographie complète sur les fouilles d'Éleusis, cf. MYLONAS, op. cit., p. 317-324 (sur les kernoi, ibid., p. 221-222).

$23 A E(1885)$, p. 174.

24 A. SKIAS, in $A E$ (1894), p. 200, n. 1, 4.

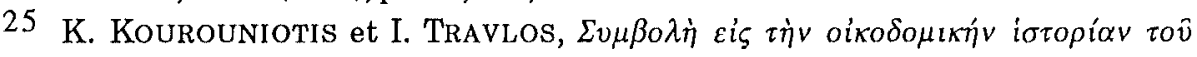

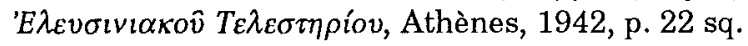

26 RUBENSOHN, art. cit., p. 303.

27 LEONARD, loc. cit. 
démonter ce dallage, étant donné que $\mathrm{D}$. Philios le tenait comme point de repère et niveau de référence pour mesurer la profondeur.

Il y a enfin, comme dernier indice chronologique, l'inscription suivante, qui figure sur un kernos en marbre (repris sur une ancienne photographie de $K$ Kourouniotis sous le $\mathrm{n}^{\circ} 10$ ), plus précisément dans la partie comprise entre l'antyx et le pied :

\section{]ATH $\Sigma$}

\section{$\triangle$ HMH]TPI KAI KOPEI} ANE@HKEN

Le dessin des lettres et surtout la forme du datif Kópeı antérieure à la réforme d'Euclide (sinon elle aurait été Kópp) indiquent la fin du Ve s. av. J.-C.

Nous avons dit au début de cet article que la kernophoria était entrée à un moment donné dans le cérémonial du culte éleusinien. Même si, à ce moment-là, la forme du kernos n'avait pas été introduite, un autre récipient aurait pu y servir : une coupe (kylix) sans pied (stemless). Et il y a à Éleusis de telles coupes décorées de motifs végétaux et de faisceaux ou d'anneaux entre les anses. La question est de savoir pourquoi, aux alentours de la fin du Ve s. av. J.-C., on introduit la forme du kernos, et pourquoi, à la fin du IVe s. av. J.-C., elle disparaît; et encore pourquoi, tandis qu'elle n'est plus employée à un moment du culte, elle continue à servir de motif décoratif et à constituer l'ornementation principale de nombreux monuments. La guerre du Péloponnèse, la période de catastrophe que vécut Athènes jusqu'à la réorganisation de beaucoup d'autres usages cultuels et religieux sous l'archontat du rhéteur Lykourgos (338-326 av. J.-C.) donneraient une bonne raison de l'introduction du kernos dans le culte à la fin du Ve s. Mais sa disparition après le IVe s. av. J.-C., aussi bien du sanctuaire d'Éleusis

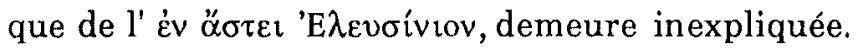

Quant à la signification de la kernophoria qui avait lieu au cours de l'initiation préalable aux Grands Mystères (qui se déroulaient, eux, au sanctuaire d'Éleusis) et qui marquait la fin de ce premier stade, elle devait être identique à celle dont étaient chargées les célébrations au cours desquelles on utilisait, ailleurs, les autres kernoi, malgré leur différence morphologique : $\pi \alpha \gamma \kappa \alpha ́ \rho \pi \varepsilon \iota \alpha \quad \sigma v \mu \mu \iota \gamma \eta \dot{\prime} \varsigma^{28}$ et $\pi \rho \circ \sigma \varphi \circ \rho \dot{\alpha}$ $\pi \alpha v \sigma \pi \varepsilon p \mu i \alpha$. L'église chrétienne orthodoxe perpétue cette sorte d'offrande

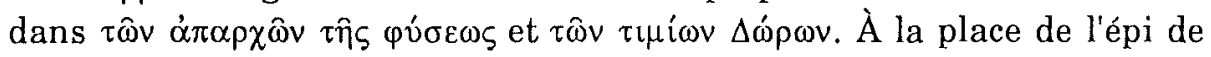

28 Fragment du Polyeus de Sophocle, cité par Clément d'AleXANDrie, Strom., IV, p. 565. Cf. PORPHYRE, De l'abstinence, II, 19. 
blé moissonné silencieusement dans la plaine thriasienne, et révélé au cours de l'épopteia à l'intérieur du Télestérion (le témoignage d'Hippolyte sur ce "mystère» vaut aussi bien pour l'initiation phrygienne que pour l'éleusinienne), à la place de la kernophoria, ce qui est aujourd'hui offert «en mémoire de Celui qui a annoncé la parole d'Amour au monde" n'est rien d'autre que quelques miettes de pain bénit, de l'eau et du vin, transsubstantiés dans un calice. L'essence du Dieu des Chrétiens est unique, et cela le distingue de ceux de l'Olympe, mais l'offrande est presque la même que celle présentée dans la kernophoria éleusinienne.

Charil. Trikoupi, 94A

G. BAKALAKIS

GR - ATHÈNES-KIFISSIA 

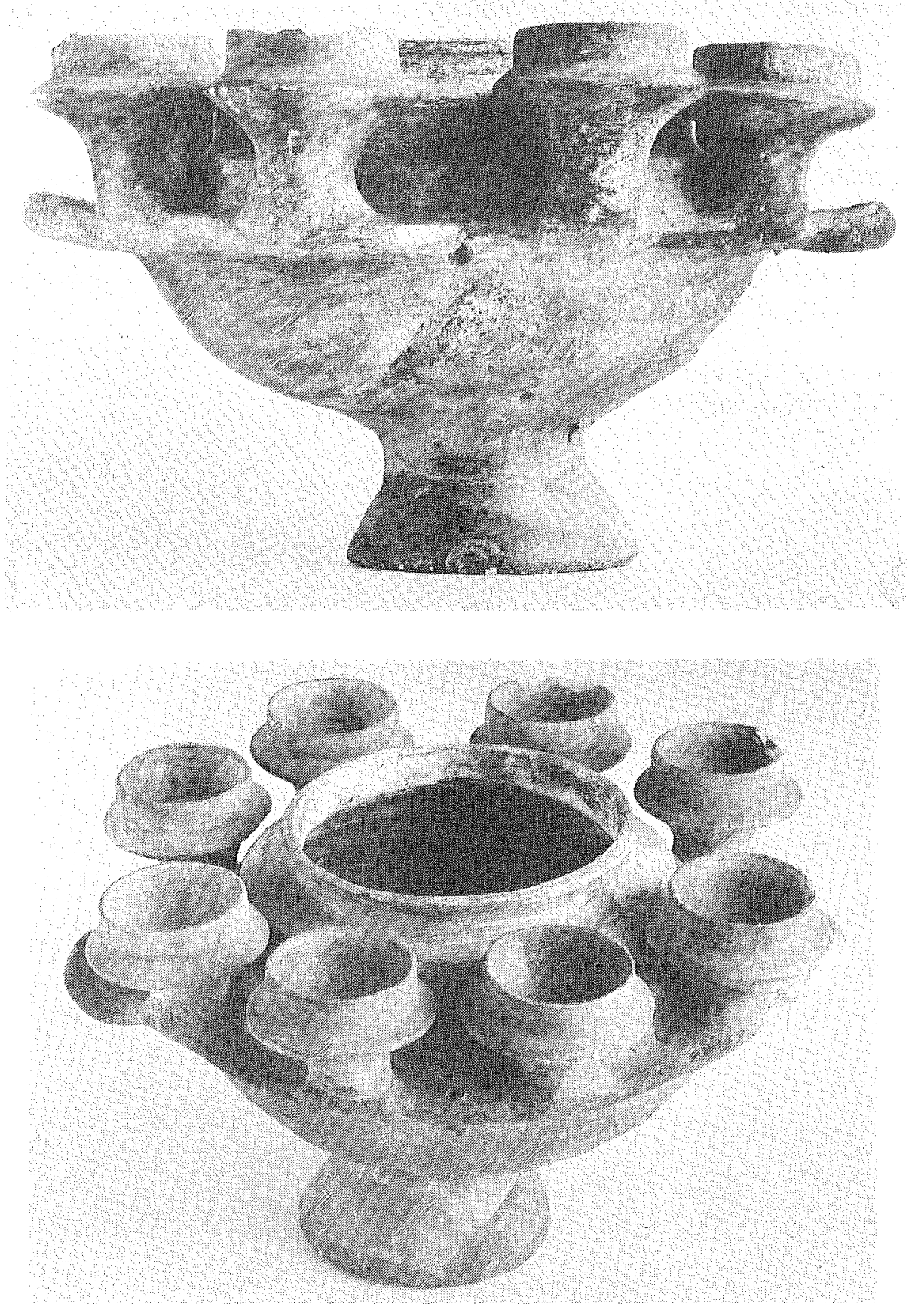

Fig. 1 : Kernos A1 (Photo : D.A.I., 71/1426-1427) 

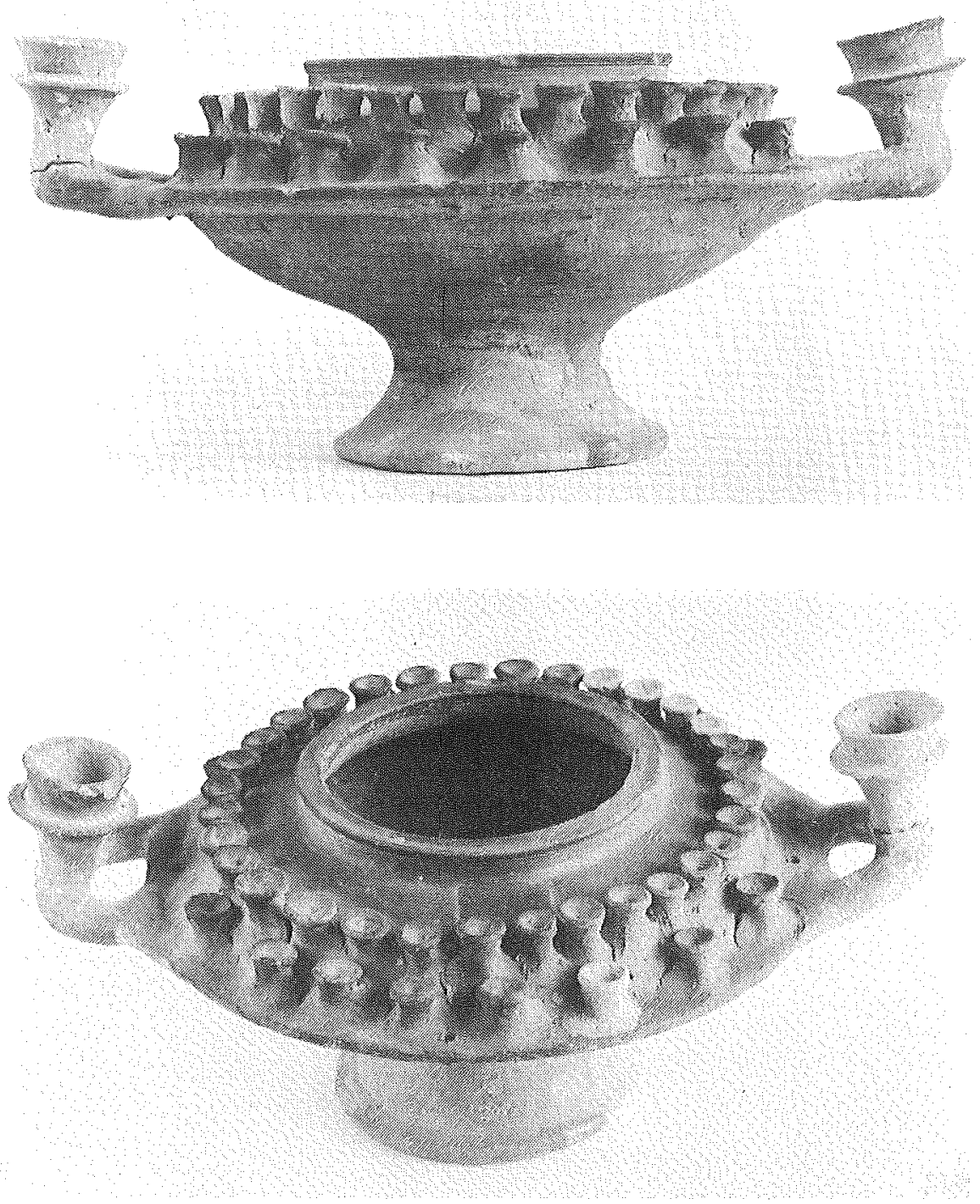

Fig. 2 : Kernos B6 (Photo : D.A.I., 71/1473-1474) 


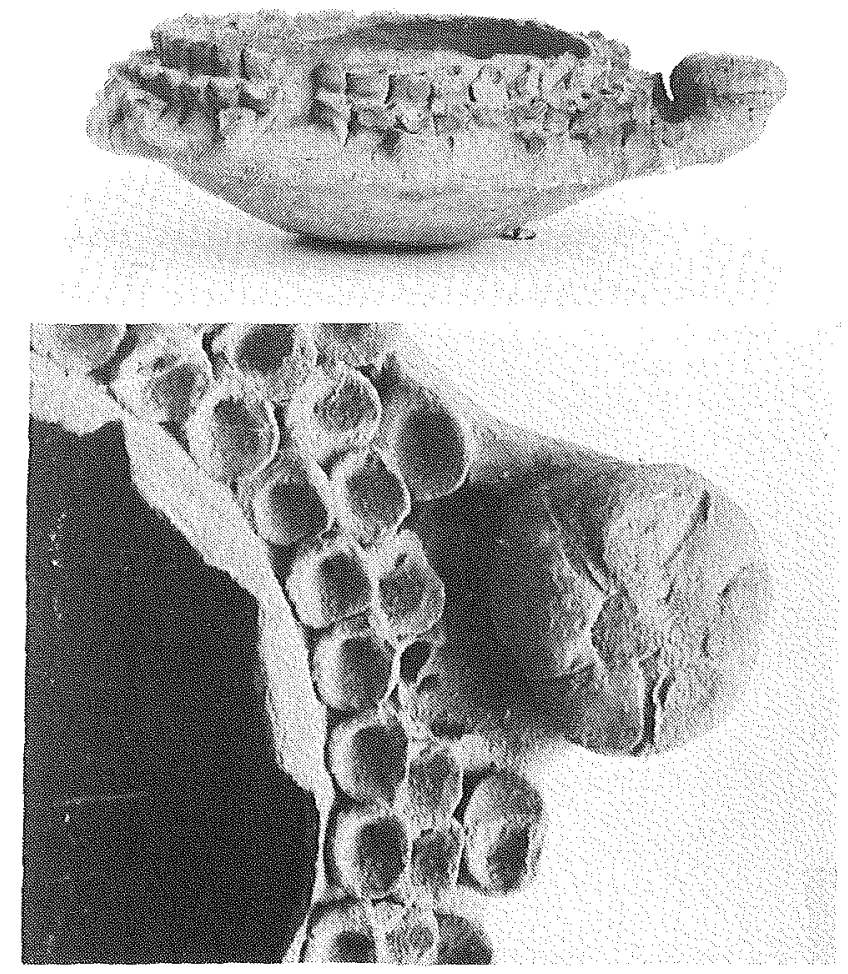

Fig. 3 : Kernos B22 (Photo : D.A.I., 71/2552)

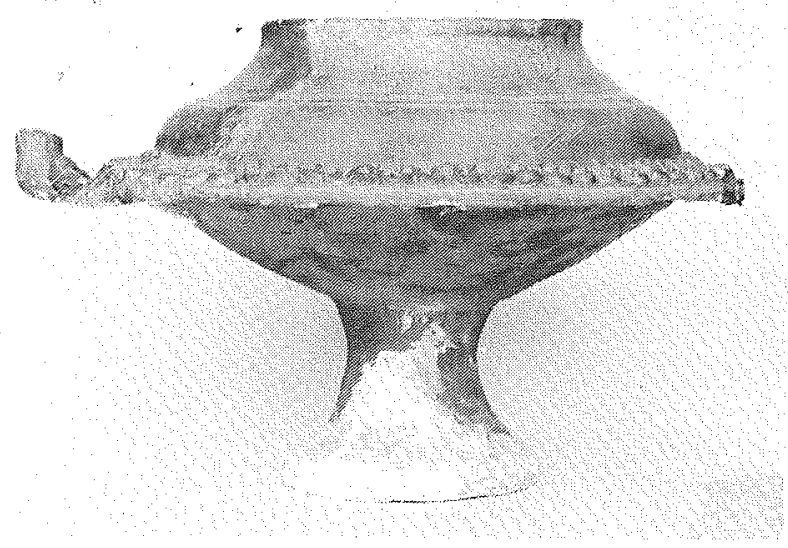

Fig. 4 : Kernos B23 (Photo : D.A.I., 71/1482) 


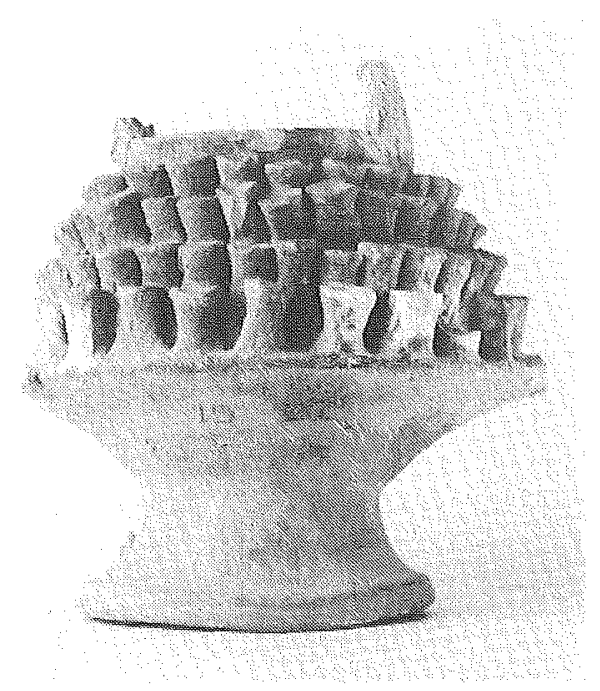

Fig. 5 : Kernos C (Photo : D.A.I., 71/1533)

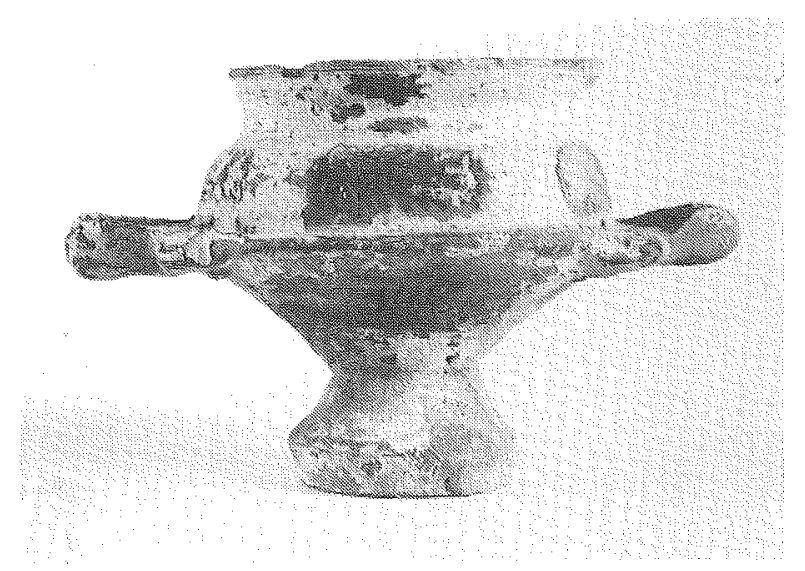

Fig. 6 : Kernos D (Photo : D.A.I., 77/1507) 